\title{
Total Pitch Specification for RHIC Helical Dipole Magnets
}

\author{
M. J. Syphers
}

July 31, 1997

In addition to the usual magnet parameter specifications, like effective length, central field, and harmonic content, the helical dipole magnets for RHIC require a specification of the effective total twist angle of the dipole field. The goal is a total twist angle of 360 degrees. With measurements of the field profile of the slotted coil design in hand - and with their excellent agreement with three dimensional calculations of this design [1] - one can model the magnet to determine the amount of twist required in the body. The procedure will be to use computer codes to model the fields given by the present end design, and vary the length (and degrees of twist) of the central field until the total field integrals $\int B_{x} d s$ and $\int B_{y} d s$ along the axis of the magnet both total zero. The question is how accurately do the fields need to be zero, or equivalently how accurately does the effective twist angle need to be 360 degrees.

A first look at this problem has been performed[2] in which it was felt that the twist requirement was very tight: $360^{\circ} \pm 0.1^{\circ}$. This came about because even small total twist errors can lead to rather large closed orbit excursions. For the Rotators, this angle gave vertical orbit errors at the tolerable limit for accelerating through spin depolarizing resonances.

However, since that time it has been realized that (a) the Rotators will be adiabatically energized at storage energy, and thus will not play a role during the acceleration through resonances, and (b) once at high energy even large (i.e., several millimeter) orbit excursions should be tolerable during storage.[3]. Thus, the criterion should be derived from tolerable vertical orbit distortions generated by two Snakes in RHIC if left uncorrected, and furthermore, one must consider reasonable local orbit corrections schemes. The effects of twist errors on the particle spin also needs to be examined. 


\section{Orbit Considerations}

\section{$1.1 \quad$ Uncorrected Orbits}

It was shown in [2] that a total twist error $2 \epsilon$ through a helical dipole magnet (which starts and ends with its field vertical) will produce trajectory errors at the end of the helix of amount

$$
\Delta\left(\begin{array}{c}
x \\
x^{\prime}
\end{array}\right)=\left(\begin{array}{c}
-\delta \epsilon \\
-2 \frac{\delta \epsilon}{L}
\end{array}\right), \quad \Delta\left(\begin{array}{c}
y \\
y^{\prime}
\end{array}\right)=\left(\begin{array}{c}
2 \delta \epsilon / \pi \\
0
\end{array}\right) .
$$

Furthermore, a collection of $N$ of these errors distributed randomly among the helical dipole magnets within the accelerator will produce closed orbit distortions with rms values

$$
\begin{aligned}
& y_{r m s} \approx \sqrt{\langle\beta\rangle \gamma_{\circ}} \frac{\delta_{r m s} \epsilon_{r m s}}{\pi|\sin \pi \nu|} \sqrt{\frac{N}{2}}, \\
& x_{r m s} \approx \frac{\delta_{r m s} \epsilon_{r m s}}{2|\sin \pi \nu|} \sqrt{1+\left(\alpha_{\circ}+2\langle\beta\rangle / L\right)^{2}} \sqrt{\frac{N}{2}},
\end{aligned}
$$

where $\delta \equiv B \ell /(k B \rho)$ for a helix, $\gamma=\left(1+\alpha^{2}\right) / \beta$ at the helices (which is constant in a lattice straight section), and $\nu$ is the betatron tune. RHIC parameters give a $0.2 \mathrm{~mm} \mathrm{rms}$ vertical orbit distortion at $250 \mathrm{GeV}$ from a total twist error of $2 \epsilon=2^{\circ}$. The corresponding horizontal orbit distortion for this same rms error, however, is about $3.5 \mathrm{~mm}$. In a Snake arrangement, where the fields begin and end vertically, it is the extra vertical fields at the ends of the magnets caused by the twist error which generate significantly larger orbit excursions in the horizontal plane.

\subsection{Local Orbit Correction}

While the $0.2 \mathrm{~mm}$ vertical distortions are tolerable, the $4 \mathrm{~mm}$ horizontal distortions caused by the Snakes will need to be corrected. Since horizontal orbit distortions do not contribute significantly to depolarizing effects, the horizontal correction certainly can be made using the usual RHIC orbit smoothing algorithms. To obtain an estimate for the required correction strength, we consider correcting orbit excursions using two local correctors at Q8 and Q10 locations, for example (assuming, for our argument, that these are horizontally focusing locations by this particular Snake). Fig. 1 schematically shows the correction scheme.

The horizontal trajectory error at the end of a single Snake is due to the individual orbit displacement errors, $\Delta$, from each helix, and the cooresponding orbital slope errors, $\theta$, propagated to the end of the Snake. Thus,

$$
\begin{aligned}
\theta_{\text {total }} & =\theta_{1}+\theta_{2}+\theta_{3}+\theta_{4} \\
\Delta_{\text {total }} & =\Delta_{1}+\Delta_{2}+\Delta_{3}+\Delta_{4}+L\left(3 \theta_{1}+2 \theta_{2}+\theta_{3}\right)
\end{aligned}
$$




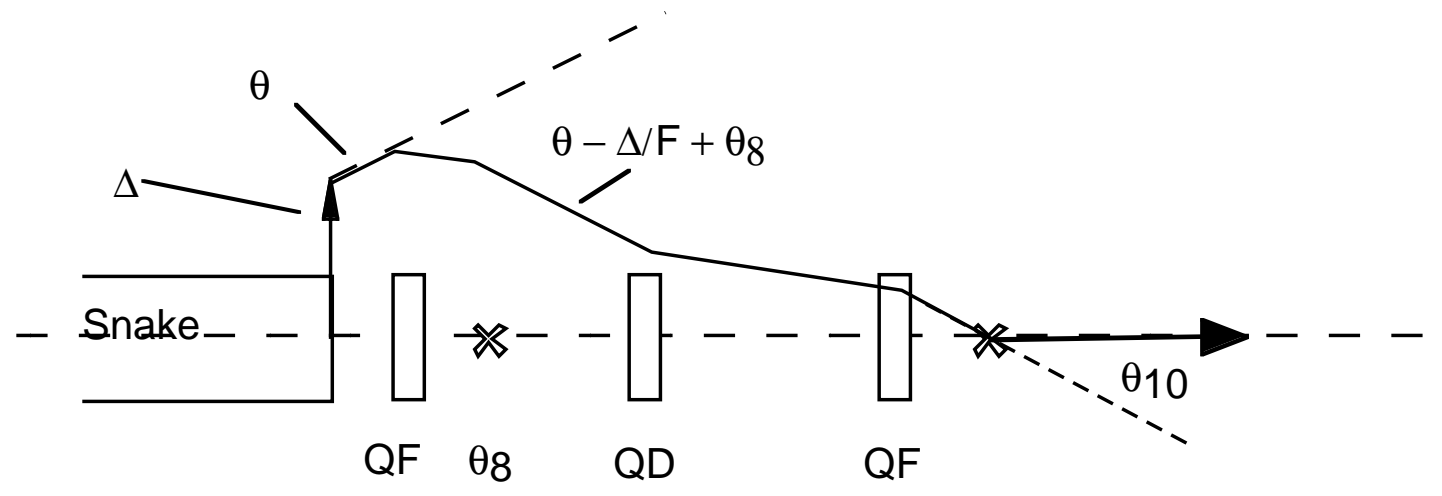

Figure 1: Orbit Correction near Snake.

where $L$ is the length of an individual helix in the Snake. For random twist errors within the Snake, the expected rms values for $\theta_{\text {total }}$ and $\Delta_{\text {total }}$ are

$$
\Delta_{r m s}=\delta_{0} \epsilon_{r m s} \sqrt{4\left[7+11\left(\frac{B_{1}}{B_{2}}\right)^{2}\right]}
$$

and

$$
\theta_{r m s}=\delta_{0} \epsilon_{r m s} \sqrt{4\left[7+11\left(\frac{B_{1}}{B_{2}}\right)^{2}\right]}
$$

where $B_{1}$ and $B_{2}$ are the field strengths $(1.25 \mathrm{~T}$ and $4 \mathrm{~T})$ of the outer and inner pairs of helices, respectively, and $\delta_{0}=B_{2} \ell /(k B \rho)$.

Assuming that the amplitude functions at the Q8 and Q10 correctors are identical and the phase advance between them is $\mu \approx 85^{\circ}$, then the corrector strengths are found from the two equations

$$
\begin{aligned}
\left(\theta_{\text {total }}-\frac{\Delta_{\text {total }}}{F}+\theta_{8}\right) \cdot\left(\cos \mu-\alpha_{0} \sin \mu\right)+\theta_{10} & =0 \\
\theta_{10} \cdot \beta_{0} \sin \mu & =\Delta_{\text {total }} .
\end{aligned}
$$

Using these last two equations, we solve for the rms values of $\theta_{8}$ and $\theta_{10}$ and obtain

$$
\begin{aligned}
\theta_{8 r m s} & =\sqrt{a^{2} \Delta_{r m s}^{2}+\theta_{r m s}^{2}-2 a\langle\theta \Delta\rangle} \\
\theta_{10 r m s} & =\frac{\Delta_{r m s}}{\beta_{\max } \sin \mu}
\end{aligned}
$$

where

$$
a \equiv \frac{1}{F}-\frac{1}{\beta_{\max } \sin \mu} \cdot \frac{1}{\cos \mu-\alpha_{0} \sin \mu}
$$




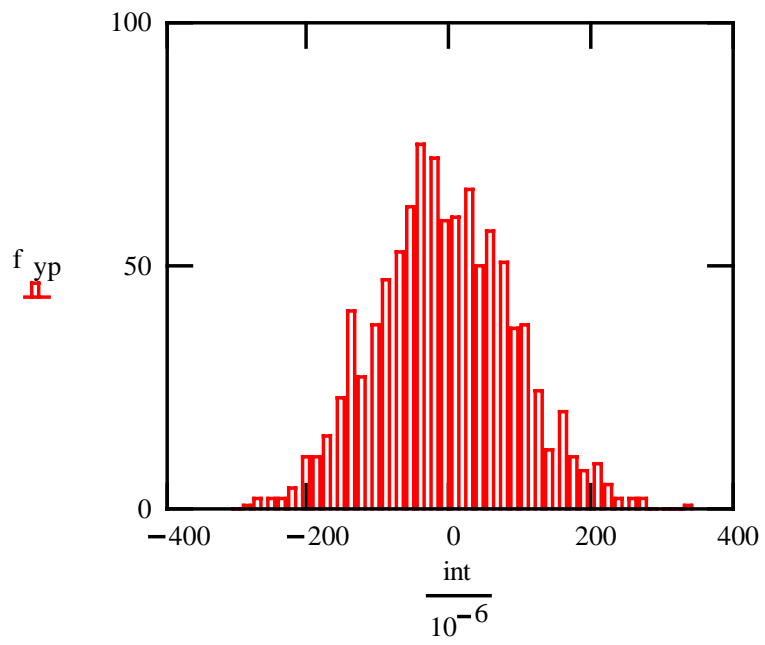

$\operatorname{stdev}(\Delta \mathrm{yp}$ end $)=97.028 \cdot 10^{-6}$

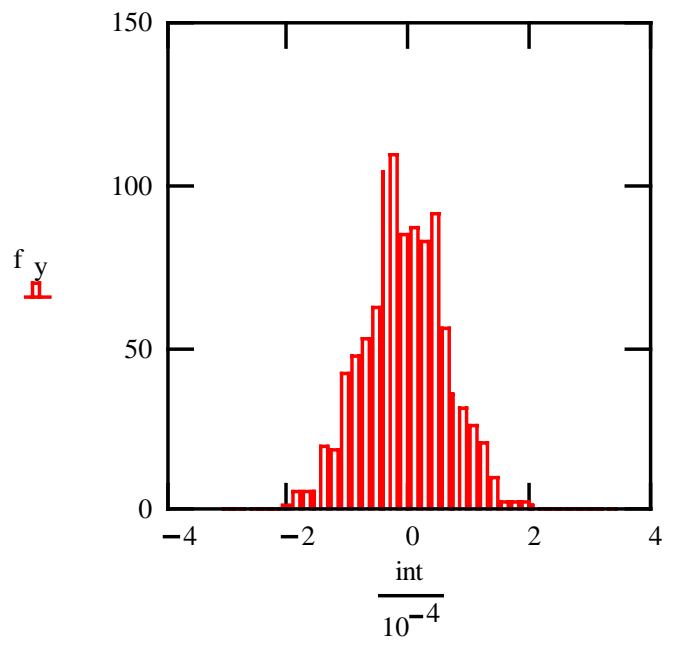

$\operatorname{stdev}(\Delta y$ end $)=0.671 \cdot \mathrm{mm}$

Figure 2: Orbit error distribution for 1000 trial Snake assemblies - angle errors (left) and position errors (right) immediately following the four helices.

and

$$
\langle\theta \Delta\rangle \equiv \frac{\left(4 \delta_{0} \epsilon_{r m s}\right)^{2}}{L} \cdot\left[1+\left(\frac{B_{1}}{B_{2}}\right)^{2}\right] .
$$

For RHIC parameters, and $2 \epsilon_{r m s}=2^{\circ}, \theta_{8 \mathrm{rms}}=43 \mu \mathrm{rad}$, and $\theta_{10 \mathrm{rms}}=10 \mu \mathrm{rad}$ at $250 \mathrm{GeV}$. At this energy, the RHIC correctors are capable of delivering $360 \mu \mathrm{rad}$ of bend strength. The above calculations are for orbit errors and their corrections at $250 \mathrm{GeV}$. Since the Snakes are kept at constant field throughout acceleration and storage, the field strengths of the steering dipoles correcting these errors would also need to be held constant.

The above correction scheme has been modeled with a Monte Carlo simulation which gives similar results. For 1000 "trial" Snake assemblies, with $2^{\circ} \mathrm{rms}$ twist errors distributed among its four helical dipole magnets, the necessary orbit corrector strengths were computed which would re-center the horizontal orbit according to the scheme described above. Fig. 2 shows the distributions in angle (left) and position (right) errors at the end of the Snake. Fig. 3 shows the distributions of the Q8 (left) and Q10 (right) corrector strengths required to correct these distortions. The results are in fair agreement with the analytical estimates.

It should also be pointed out that a residual twist angle of 2 degrees is equivalent to a transverse field integral of $\int B_{y} d s=2 \epsilon_{r m s} B_{2} / k_{i d e a l}=533$ gauss-meters. 


$$
\operatorname{stdev}\left(\theta_{8}\right)=32.504 \cdot 10^{-6}
$$

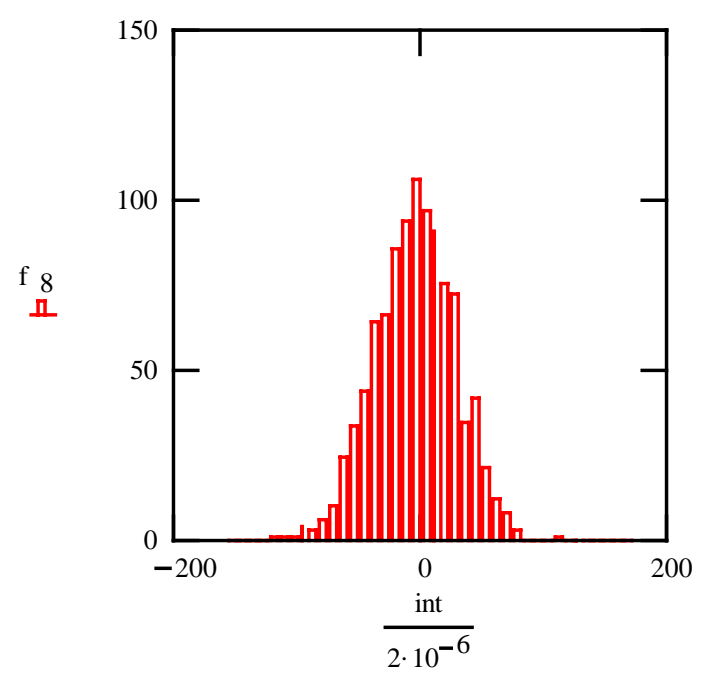

$\operatorname{stdev}\left(\theta_{10}\right)=14.966 \cdot 10^{-6}$

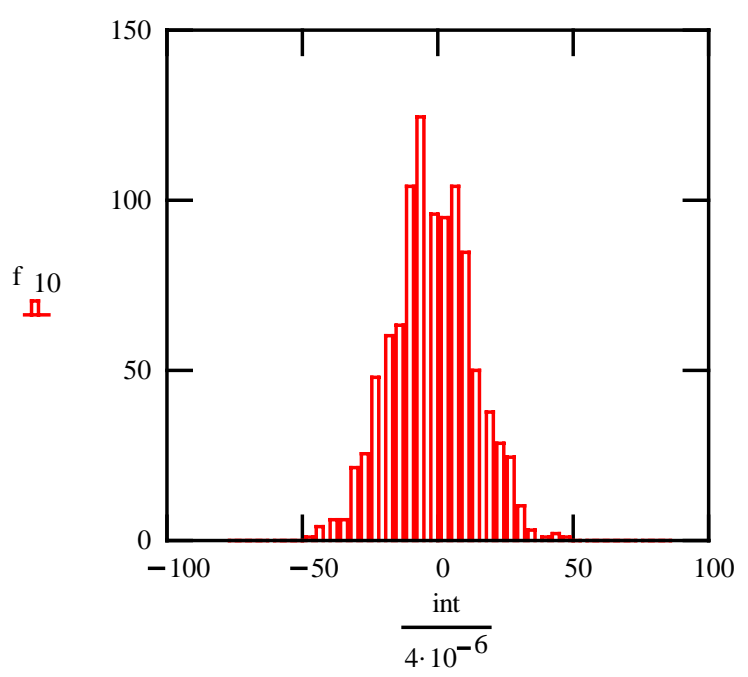

Figure 3: Corrector distribution for the same 1000 trial Snake assemblies using Q8 (left) and Q10 (right) steering dipole correctors.

\section{Spin Considerations}

One would not expect the small, localized orbit distortions and their corrections to have much effect on the spin behavior of the proton beams. To verify this statement, a spin vector was tracked for 2000 revolutions about each of the 1000 trial accelerators. An "accelerator" was assumed to consist of two Snakes, one of which had the twist errors from the trials above, and the other which was assumed perfect. The horizontal orbit distortions from the Snake with twist errors were corrected with the Q8 and Q10 correctors, and the effects of these correctors (vertical fields) on the spin were accounted for. For each trial, the initial spin vector was assumed vertical, and the distribution of the vertical component of the resultant spin vectors after 2000 revolutions is shown in Fig. 4. As can be seen in this figure, the worst cases show about a $0.5 \%$ change in the vertical spin component, but most of the cases are well within $0.1 \%$ of the initial vertical spin direction after 2000 revolutions. The result appeared to be qualitatively independent of the number of revolutions tracked.

The resonance strength of the twist errors can be computed by looking at the effect of the errors as a perturbation to the nominal spin motion. To do so, the following technique was used. For each of the 1000 trials, the spin matrix for the orbit-corrected Snake was computed. Let the matrices for the helices with errors be $M_{\epsilon, 1}, M_{\epsilon, 2}, M_{\epsilon, 3}$, and $M_{\epsilon, 4}$, and the matrices for the correctors $M_{8}, M_{10}$. In addition, the spin is affected by the offsets in the quadrupoles Q8 and Q9 (we assume the correctors center the orbit in Q10) through the matrices $M_{q 8}$ and $M_{q 9}$, and is also 


$$
\begin{aligned}
\operatorname{stdev}\left(\mathrm{S}_{\mathrm{y}}\right)=1.346 \cdot 10^{-3} \quad \operatorname{median}\left(\mathrm{S}_{\mathrm{y}}\right) & =0.99949 \\
\operatorname{mean}\left(\mathrm{S}_{\mathrm{y}}\right) & =0.99898
\end{aligned}
$$

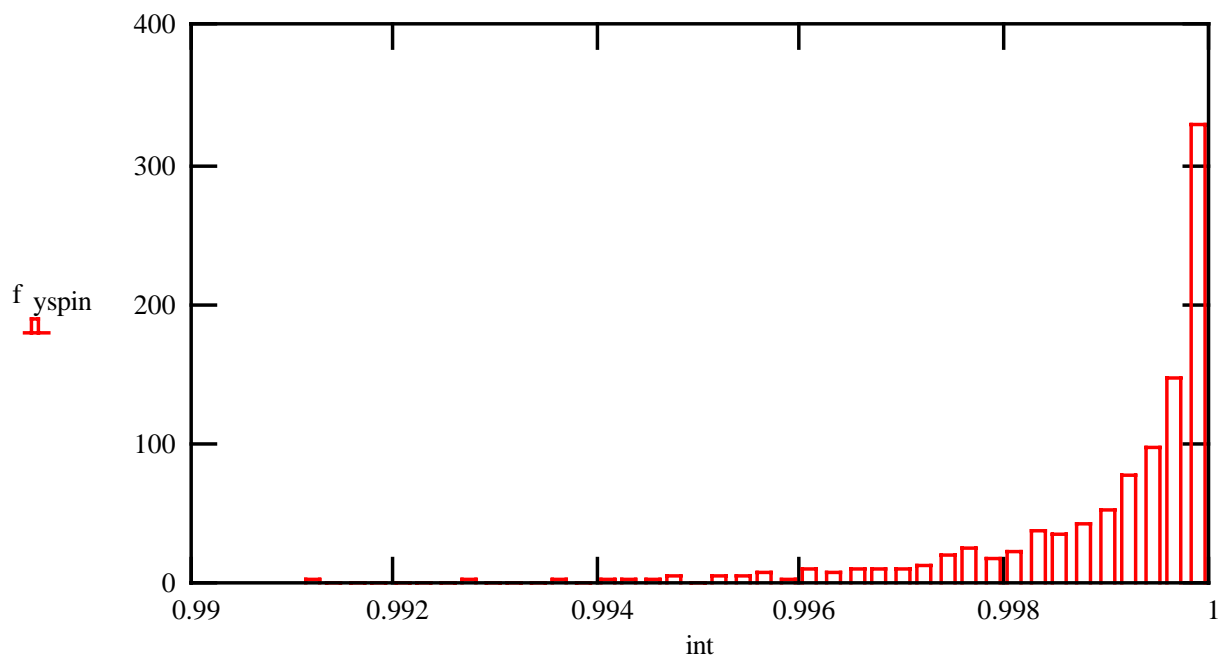

Figure 4: Distsribution of vertical spin component after 2000 revolutions for the 1000 trial accelerators. For each trial, the initial spin vector was vertical.

affected by the two RHIC bending magnets within this region via $M_{B}$. Then the total spin matrix for the corrected Snake is thus

$$
M_{T}=M_{10} M_{B} M_{q 9} M_{B} M_{8} M_{q 8} M_{\epsilon, 4} M_{\epsilon, 3} M_{\epsilon, 2} M_{\epsilon, 1} .
$$

We define the perturbation matrix $\Delta M$ as

$$
\Delta M=M_{0,1}^{-1} M_{0,2}^{-1} M_{0,3}^{-1} M_{0,4}^{-1} M_{B}^{-1} M_{B}^{-1} M_{T}
$$

where here, $M_{0, j}$ is the matrix for the $j$-th helical dipole in the Snake without errors. Thus, $\Delta M$ generates the contribution to the spin motion just due to the twist errors in the helical dipole magnets. It reduces to the unit matrix as the twist errors go to zero.

Now, if a vertically directed spin vector is propogated through this perturbation matrix $\Delta M$, then the angle between the resultant vector and the vertical direction will be the amount of rotation per turn about a horizontal axis generated by the perturbation. The resonance strength is then given by this angle divided by $2 \pi$.

The resonance strength due to a single Snake in RHIC with $2^{\circ}$ rms twist errors and a corrected horizontal orbit was calculated for the 1000 trials presented above. The results are shown in the histogram of Fig. 5. The typical resonance strength was roughly $\epsilon_{\text {res }} \approx 0.008$, and for $95 \%$ of the trials $\epsilon_{\text {res }}$ was less than 0.015 . The spec- 


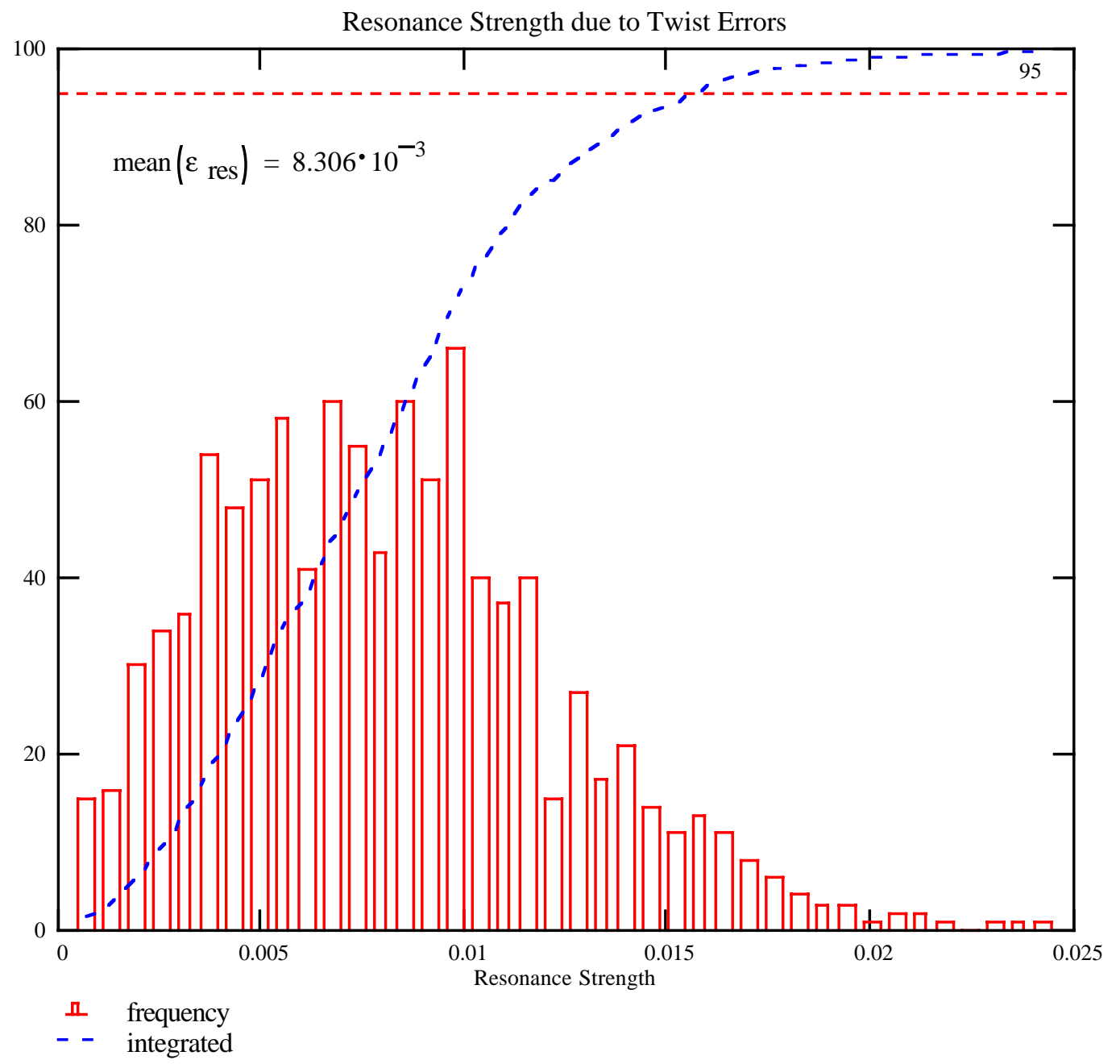

Figure 5: Distsribution of resonance strengths due to twist errors in a single Snake for the 1000 trial accelerators. 
ification for polarized beam operation in RHIC is that total imperfection resonance strengths be less than 0.05.[4]

\section{Conclusions}

From the analysis of orbit distortions and spin behavior due to twist errors in the helical dipole magnets, it appears that a total twist angle of $360^{\circ} \pm 2^{\circ}$ is easily tolerable in RHIC so long as the Spin Rotators are not energized during acceleration. Without local correction, the orbit distortions from two Snakes in RHIC should give tolerable (less than $0.2 \mathrm{~mm} \mathrm{rms}$ at high field) orbit excursions in the vertical plane. While the horizontal orbit excursions would be significantly larger (several $\mathrm{mm}$ ), they are easily corrected with the RHIC dipole correction system. The effect on the particle spin from the twist errors and from the horizontal correctors is tolerable.

Since the total integrated "twist angle" is somewhat hard to define in a magnet with real ends, it is better to set the requirement in terms of the integrated field components through the helical dipole. A twist angle of $360^{\circ} \pm 2^{\circ}$ in this regard is equivalent to requiring $\left|\int B_{x, y} d \ell\right|<500$ Gauss-meter for a central field of 4.0 Tesla and an effective magnetic length of $2.4 \mathrm{~m}$.

\section{References}

[1] M.Okamura, T. Kawaguchi, T. Tominaka, T. Katayama, "Three Dimensional Field Analysis of Helical Magnet for RHIC Siberian Snake," proc. 1997 Part. Accel. Conf., Vancouver, to be published.

[2] M.J. Syphers, AGS/RHIC/SN-016, Dec. 1995.

[3] A. Luccio, Private Communication.

[4] "Preliminary Design Report - Polarized Proton Collider at RHIC," BNL report to be published. 